\title{
Successful use of extracorporeal membrane oxygenation during cesarean section in parturients with symptomatic severe pulmonary stenosis: a case report
}

\author{
Doo-Hwan Kim, Gi-Ho Koh, Sang-A Lee, Young-Kug Kim, Dong-Min Jang \\ Department of Anesthesiology and Pain Medicine, Asan Medical Center, University of Ulsan College of Medicine, Seoul, Korea
}

\author{
Received January 10, 2019 \\ Revised April 9, 2019 \\ Accepted April 10, 2019 \\ Corresponding author \\ Dong-Min Jang \\ Department of Anesthesiology \\ and Pain Medicine, Asan Medical \\ Center, University of Ulsan College \\ of Medicine, 88, Olympic-ro 43-gil, \\ Songpa-gu, Seoul 05505, Korea \\ Tel: +82-2-3010-5308 \\ Fax: +82-2-3010-6790 \\ E-mail: dmjang@amc.seoul.kr \\ ORCID: \\ https://orcid.org/0000-0002-6254-9467
}

\begin{abstract}
Optimizing anesthetic management during cesarean section in parturients with severe pulmonary stenosis is difficult. This report describes the use of extracorporeal membrane oxygenation (ECMO) in a patient with symptomatic severe pulmonary stenosis undergoing cesarean section. A 33-year-old primigravid woman was admitted to the emergency department during the $37^{\text {th }}$ week of gestation for vaginal leakage and dyspnea on exertion. Transthoracic echocardiography showed severe pulmonary valve stenosis with a maximal gradient of $135 \mathrm{mmHg}$, combined with right ventricular hypertrophy and moderately decreased right ventricular contractility. She underwent emergency cesarean section under general anesthesia with ECMO as an adjuvant anesthetic method. Hemodynamic variables were well-maintained in the perioperative period, and the baby was delivered without complications. The patient was successfully weaned off of the ECMO device 3 hours after the end of the operation. The mother and baby were discharged in good health on hospital day 6. General anesthesia with ECMO support can be safe and effective in patients with severe pulmonary stenosis undergoing cesarean section.
\end{abstract}

Keywords: Cesarean section; Extracorporeal membrane oxygenation; Pregnant woman; Pulmonary valve stenosis; General anesthesia

\section{INTRODUCTION}

Severe pulmonary stenosis in parturients is rare, but may be fatal [1]. To date, no anesthetic method has been found optimal for pregnant women with cardiac valve disease undergoing cesarean section. Therefore, managing anesthesia in parturients with severe pulmonary stenosis is difficult. Extracorporeal membrane oxygenation (ECMO) support devices are widely used to manage patients with cardiopulmonary failure. ECMO can be used as an adjuvant to anesthetize critically ill patients with high-risk cardiopulmonary or airway disease [2-4]. This report demonstrates the successful and safe utilization of ECMO in a parturient with severe pulmonary stenosis undergoing cesarean section.

\section{CASE REPORT}

A 33-year-old primigravid parturient was admitted to the emergency department in the $37^{\text {th }}$ week of gestation for vaginal leakage and dyspnea on exertion (DOE). At age 5, she had undergone total correction of tetralogy of Fallot. Examination in the obstetric outpatient department in the $18^{\text {th }}$ week of gestation showed shortness of breath, categorized as New York Heart Association (NYHA) class II, which 
later increased to NYHA class III at admission. A grade 4/5 systolic murmur was detected at the left upper sternal border. Bilateral leg edema was noted, and chest X-rays showed cardiomegaly.

The initial vital signs included a blood pressure of 101/71 $\mathrm{mmHg}$, pulse rate of 97 beats per minute, and respiratory rate of 25 breaths per minute. Twelve-lead electrocardiography showed sinus rhythm with premature ventricular complexes and right axis deviation with right ventricular hypertrophy. Transthoracic echocardiography (TTE) revealed severe pulmonary valve stenosis. The maximal transvalvular pressure gradient was $135 \mathrm{mmHg}$. The right atrium was enlarged, and the right ventricle was hypertrophic, with moderately decreased right ventricular contractility. Right ventricular compression of the ventricular septum toward the left ventricle resulted in a D-shaped left ventricle (Fig. 1). Inferior vena cava plethora was also observed. Arterial blood tests after oxygen supplementation with a simple mask at $6 \mathrm{~L} / \mathrm{min}$ showed: $\mathrm{pH}, 7.51 ; \mathrm{PaCO}_{2}, 28 \mathrm{mmHg} ; \mathrm{PaO}_{2}, 98$ $\mathrm{mmHg}$; and base excess, $-0.3 \mathrm{mM}$. Other blood tests were within normal ranges, except the hemoglobin concentration, which was $8.6 \mathrm{~g} / \mathrm{dL}$.

The baby was in a breech position, and amniotic fluid was leaking from the mother's vagina because of premature rupture of membranes. After consulting and discussing with the cardiac surgeon, emergency cesarean section under general anesthesia with ECMO (Capiox Emergency Bypass System; Terumo Inc., Tokyo, Japan) support was indicated.

In the operating room, a cannula was inserted in the left radial artery to monitor arterial blood pressure continuously. After placing a central venous catheter into the right internal jugular vein, the central venous pressure was 29
$\mathrm{mmHg}$, which correlated with inferior vena cava plethora on preoperative TTE (Fig. 2). The femoral vein and artery were sequentially cannulated with $21-\mathrm{Fr}$ cannulas under ultrasound guidance by an ECMO specialist. The venous cannula tip was positioned at the junction of the right atrium and the inferior vena cava, as confirmed on TTE. ECMO support was started, resulting in an immediate and dramatic reduction in central venous pressure, along with a slight reduction in blood pressure. As postpartum hemorrhage may be life-threatening, anticoagulants were not administered in the perioperative period. An alternative procedure consists of short-term ECMO, followed by early weaning from ECMO to prevent thromboembolic events.

After verifying the maintenance of ECMO flow, general anesthesia was induced with $250 \mathrm{mg}$ of thiopental sodium and $80 \mathrm{mg}$ of rocuronium. Rapid sequence induction (RSI) was performed with Sellick's maneuver. For maintaining hemodynamic stability during RSI, remifentanil was administrated via target-controlled infusion using Orchestra (Fresenius Vial, Brezins, France). Anesthesia was maintained with 0.7 minimum alveolar concentration of sevoflurane in an air/ $\mathrm{O}_{2}$ mixture and an effect-site remifentanil concentration of $2 \mathrm{ng} / \mathrm{mL}$. ECMO flow was maintained between 3.0 and $4.0 \mathrm{~L} / \mathrm{min}$. Although advancing a Swan-Ganz catheter in the pulmonary artery failed because of severe pulmonary valve stenosis, the right ventricular pressure increased, becoming similar to the left ventricular systolic pressure (Fig. 2).

When performing the incision of cesarean section, the effect-site remifentanil concentration was increased to 5 $\mathrm{ng} / \mathrm{mL}$ to alleviate the activation of the sympathetic nervous system, and ECMO support effectively decompressed the right ventricular pressure and work to prevent right
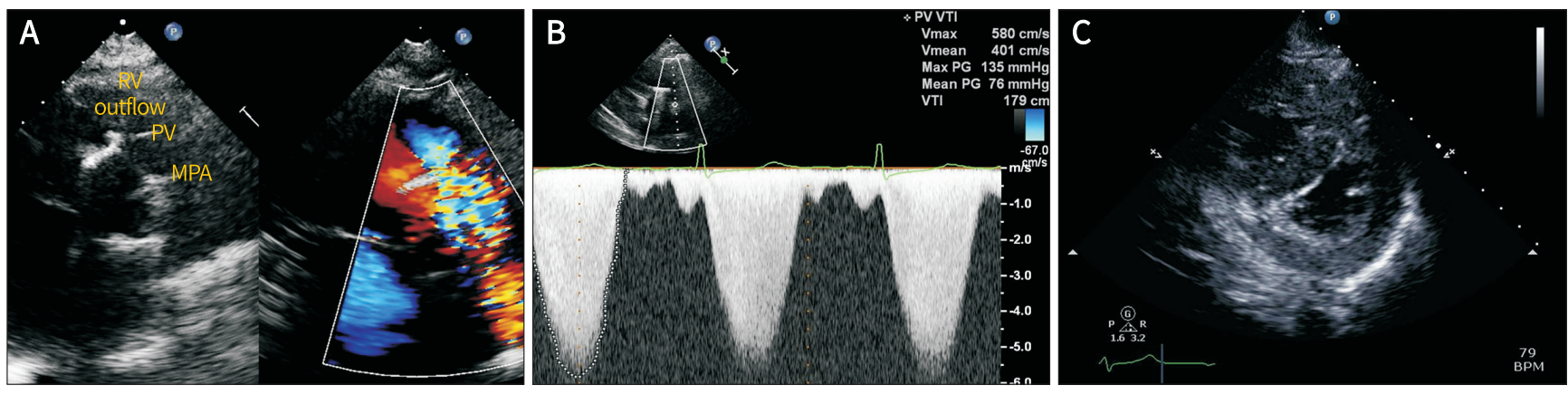

Fig. 1. Ultrasound results of our patient. (A) Severe pulmonary valve stenosis on 2D and color Doppler images. (B) Calculated maximal transvalvular pressure gradient on continuous wave Doppler image. (C) D-shaped left ventricle on parasternal short axis view before extracorporeal membrane oxygenation insertion. 


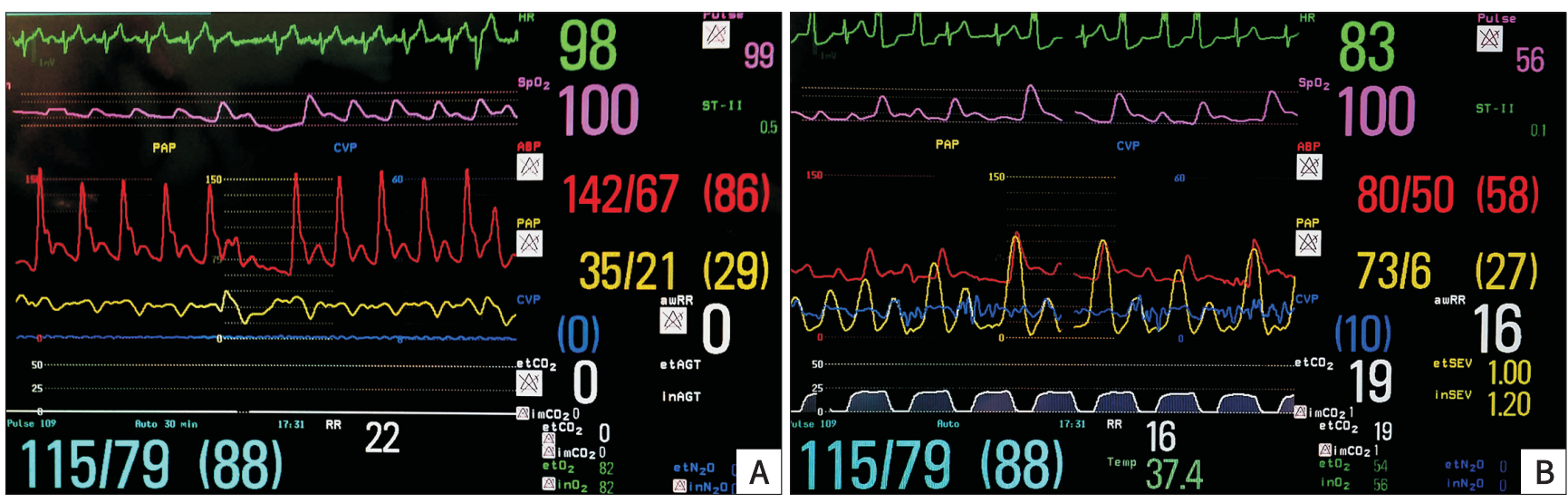

Fig. 2. Vital signs in our patient. (A) Development of high central venous pressure after central line insertion. (B) Following placement of the tip of the SwanGanz catheter in the right ventricle, right ventricular systolic pressure increased to a level similar to that of left ventricular systolic pressure.

ventricular dysfunction from incision-induced sympathetic stimulation. We tried to maintain ECMO flow at 3.0-4.0 L/ min. Volume replacement was appropriately performed to replace blood loss and prevent hypotension. Blood pressure was continuously monitored, and the targeted mean blood pressure was 60-70 mmHg. Cesarean section was successfully completed. Hemodynamic variables were well-maintained throughout the perioperative period using ECMO support. A healthy female neonate was delivered with Apgar scores of 8 at 1 minute and 9 at 5 minutes. The patient was infused with $1.3 \mathrm{~L}$ of crystalloid solution and $500 \mathrm{~mL}$ of $5 \%$ albumin, and was transfused with 4 units of packed red blood cells. The estimated blood loss was about 1.0 L. In the postoperative period, the patient was cared for in the intensive care unit. To avoid hypervolemic or hypovolemic status after surgery, hemodynamic parameters of the patient and the ECMO device were delicately monitored, and the targeted ECMO flow (3.0-4.0 L/min) and mean blood pressure (60-70 mmHg) were maintained. The patient was uneventfully weaned from the ECMO device 3 hours after the end of the operation.

The patient and baby were discharged without complications on hospital day 6 . Three months later, the patient underwent pulmonary valve replacement because of infective endocarditis.

\section{DISCUSSION}

About $10 \%$ of patients who undergo complete repair of tetralogy of Fallot as children later develop severe pulmonary stenosis [5]. Although parturients with severe pulmonary stenosis are considered an intermediate-risk group, those with NYHA class III or IV functional capacity should be regarded as a high-risk group [1]. Therefore, it is necessary to understand the pathophysiology of pulmonary valve stenosis and it is affected by pregnancy.

Pulmonary stenosis leads to obstruction of the right ventricular outflow, increasing right ventricular pressure and work. Sustained high pressure on the right ventricle induces right ventricular hypertrophy. Chronic overloading of the right ventricle can cause right-sided cardiac failure. Several types of cardiovascular changes result in the deterioration of right ventricular function in parturients. First, intravascular volume is approximately $40 \%$ higher in pregnant women than in non-pregnant women. This excess preload can aggravate right ventricular dysfunction. Second, increase in stroke volume and heart rate can increase cardiac output by approximately $40-50 \%$, and inordinate increase in cardiac output may induce acute cardiac decompensation. Therefore, stable and safe hemodynamic management in women with severe pulmonary stenosis undergoing cesarean section requires maintenance of proper preload, heart rate within normal ranges, and prevention of reduced afterload and myocardial contractility. However, these steps remain difficult, as no consensus has yet been reached on the anesthetic management of patients with severe pulmonary stenosis.

Several reports describe anesthetic experiences of patients with pulmonary stenosis undergoing vaginal delivery or cesarean section [6-9]. The use of epidural anesthesia in a parturient with pulmonary stenosis undergoing cesarean 
section resulted in marked hypotension, junctional arrhythmia, and chest pain. However, the patient and infant were healthy and had no complications after delivery [6]. Cesarean section was performed using combined spinal and epidural anesthesia in a patient with severe pulmonary stenosis and a peak systolic gradient of $85 \mathrm{mmHg}$ [7]. Hemodynamic parameters were well-maintained, and a healthy infant was delivered. A patient with severe pulmonary stenosis (peak gradient: $68 \mathrm{mmHg}$ ) successfully underwent cesarean section under general anesthesia using a mixture of isoflurane and air $/ \mathrm{O}_{2}$, with both the mother and baby being healthy and discharged uneventfully [8].

ECMO is mostly used for cardiopulmonary support in patients with cardiogenic shock or respiratory failure. Traditionally, ECMO has been utilized for life-threatening conditions in the intensive care unit but was recently found useful as an adjuvant to anesthetize critically ill patients with highrisk cardiopulmonary (acute respiratory distress syndrome, end-stage lung disease requiring lung transplantation, or cardiogenic shock) or airway disease (tracheal stenosis or obstruction) in the operating room [2-4]. Several reports describe the use of ECMO in pregnancy [10-14], mostly for acute respiratory distress due to pneumonia or influenza infection, although only a few case reports describe the use of ECMO as an adjunct to anesthesia during cesarean section [11,15].

Although the severity of pulmonary stenosis was found to have little effect on maternal and fetal outcomes [16], the maximal pressure gradient of $135 \mathrm{mmHg}$ in this patient was high. Furthermore, she had right ventricular dysfunction and DOE (NYHA class III) combined with pulmonary stenosis. As morbidity and mortality in pregnant women with underlying cardiac disease have shown strong correlations with the functional status [1], our patient was regarded as being high-risk. Moreover, in a case report, a parturient with severe pulmonary hypertension (maximal pressure gradient of $106 \mathrm{mmHg}$ ) underwent cardiogenic shock during cesarean section [17]. Therefore, after consulting a cardiac surgeon, we determined an emergency cesarean section under general anesthesia with ECMO support in this patient.

The combination of ECMO and general anesthesia has been used in the perioperative period for anesthetic management of patients with poor cardiac tolerance. Importantly, surgical stresses, including incision and surgical bleeding, may be critical for a parturient with severe pulmonary stenosis during the cesarean section. For maintaining hemodynamic stability and reducing cardiac stress, ECMO flow should be maintained at $80 \%$ or more of the cardiac output even if a vasopressor or volume replacement (fluid or blood products) is used [18]. ECMO effectively alleviates right ventricular pressure and work when the sympathetic nervous system is activated, resulting in reduced cardiac work. Moreover, it can maintain appropriate blood pressure in cases of intraoperative bleeding or hypotension, thereby preventing right ventricular dysfunction from perioperative stress. ECMO maintained hemodynamic stability and prevented acute cardiac decompensation, suggesting that general anesthesia combined with ECMO could be better than other anesthetic methods for patients with severe pulmonary stenosis.

There are some concerns regarding ECMO support during cesarean section. The most common complication in patients on ECMO is bleeding. Anticoagulants, such as heparin, can induce intractable postpartum bleeding, which can lead to hemodynamic instability and coagulopathy, and occasionally death. Consequently, anticoagulants were not administered in our patient. Lack of anticoagulants may cause thromboembolism, which may result in a catastrophic event. However, short-term use of ECMO without anticoagulation reduced the rates of blood transfusion and risks of bleeding without increasing the risk of thromboembolism [19]. In the absence of systemic anticoagulation in our patient, heparin-coated circuits and a membrane oxygenator were utilized to prevent thromboembolism. ECMO was performed for 4 hours, and the patient was uneventfully weaned from the ECMO device. Other reports on the use of ECMO without anticoagulants showed similar results as ours [19-21]. Another major concern may be the effect of ECMO on the fetus. During ECMO support in pregnancy, most fetuses survived [22]. A few fetuses died in cases of maternal death or fetal hypoperfusion.

ECMO can provide delicate adjuvant anesthetic management and maintain hemodynamic stability in the perioperative period, especially in high-risk parturients with cardiopulmonary disease. These findings suggest that general anesthesia with ECMO support is safe and effective in patients with severe pulmonary stenosis and right ventricular dysfunction undergoing cesarean section. 


\section{ACKNOWLEDGEMENTS}

The authors thank Dr. Ju Yong Lim (cardiac surgeon) in the Department of Thoracic and Cardiovascular Surgery, Seoul St Mary's Hospital, for insertion and management of ECMO in this case.

\section{CONFLICT OF INTEREST}

No potential conflict of interest relevant to this article was reported.

\section{REFERENCES}

1. Dob DP, Yentis SM. Practical management of the parturient with congenital heart disease. Int J Obstet Anesth 2006;15:137-44

2. Connolly KM, McGuirt WF Jr. Elective extracorporeal membrane oxygenation: an improved perioperative technique in the treatment of tracheal obstruction. Ann Otol Rhinol Laryngol 2001;110:205-9.

3. Huang SC, Wu ET, Chi NH, Chiu SN, Huang PM, Chen YS, et al. Perioperative extracorporeal membrane oxygenation support for critical pediatric airway surgery. Eur J Pediatr 2007;166:1129-33.

4. Odonkor PN, Stansbury L, Garcia JP, Rock P, Deshpande SP, Grigore AM. Perioperative management of adult surgical patients on extracorporeal membrane oxygenation support. J Cardiothorac Vasc Anesth 2013;27:329-44.

5. Brickner ME, Hillis LD, Lange RA. Congenital heart disease in adults. First of two parts. N Engl J Med 2000;342:256-63.

6. Campbell N, Rosaeg OP, Chan KL. Anaesthetic management of a parturient with pulmonary stenosis and aortic incompetence for Caesarean section. Br J Anaesth 2003;90:241-3.

7. Makkar JK, Jindal S, Jain A, Wig J. Anaesthetic management of a parturient with severe pulmonary stenosis undergoing Caesarean section. S Afr J Anaesthesiol Analg 2010;16:28-30.

8. Sanikop CS, Umarani VS, Ashwini G. Anaesthetic management of a patient with isolated pulmonary stenosis posted for caesarean section. Indian J Anaesth 2012;56:66-8.

9. Ransom DM, Leicht $\mathrm{CH}$. Continuous spinal analgesia with sufentanil for labor and delivery in a parturient with severe pulmonary stenosis. Anesth Analg 1995;80:418-21.

10. Cunningham JA, Devine PC, Jelic S. Extracorporeal membrane oxygenation in pregnancy. Obstet Gynecol 2006;108: 792-5.
11. Di Lorenzo G, Martucci G, Sciacca S, Longo R, Pilato M, Arcadipane A. Dysfunction of mechanical mitral prosthesis at 33rd week of pregnancy: ECMO support as a complex strategy for the mother and the fetus. Perfusion 2016;31:611-3.

12. Lysenko L, Zaleska-Dorobisz U, Blok R, Dumański A, Zielińska $\mathrm{M}$, Kustrzycki W, et al. A successful cesarean section in a pregnant woman with $\mathrm{A}(\mathrm{H} 1 \mathrm{~N} 1)$ influenza requiring ECMO support. Kardiochir Torakochirurgia Pol 2014;11:216-9.

13. Panarello G, D’Ancona G, Capitanio G, Occhipinti G, Attardo G, Bertani A, et al. Cesarean section during ECMO support. Minerva Anestesiol 2011;77:654-7.

14. Robertson LC, Allen SH, Konamme SP, Chestnut J, Wilson $P$. The successful use of extra-corporeal membrane oxygenation in the management of a pregnant woman with severe H1N1 2009 influenza complicated by pneumonitis and adult respiratory distress syndrome. Int J Obstet Anesth 2010;19:443-7.

15. Sugioka J, Nakajima T, Ohsumi H, Kuro M, Sasako Y. [Anesthetic management using percutaneous cardiopulmonary support for cesarean section in a patient with severe pulmonary hypertension]. Masui 1995;44:574-8. Japanese.

16. Hameed AB, Goodwin TM, Elkayam U. Effect of pulmonary stenosis on pregnancy outcomes--a case-control study. Am Heart J 2007;154:852-4

17. Hara R, Hara S, Ong CS, Schwartz G, Sciortino C, Hibino N. Cesarean section in the setting of severe pulmonary hypertension requiring extracorporeal life support. Gen Thorac Cardiovasc Surg 2017;65:532-4.

18. Choi JH, Kim SW, Kim YU, Kim SY, Kim KS, Joo SJ, et al. Application of veno-arterial-venous extracorporeal membrane oxygenation in differential hypoxia. Multidiscip Respir Med 2014;9:55.

19. Lamarche Y, Chow B, Bédard A, Johal N, Kaan A, Humphries $\mathrm{KH}$, et al. Thromboembolic events in patients on extracorporeal membrane oxygenation without anticoagulation. Innovations (Phila) 2010;5:424-9.

20. Arlt M, Philipp A, Voelkel S, Rupprecht L, Mueller T, Hilker M, et al. Extracorporeal membrane oxygenation in severe trauma patients with bleeding shock. Resuscitation 2010;81:8049.

21. Wen PH, Chan WH, Chen YC, Chen YL, Chan CP, Lin PY. Non-heparinized ECMO serves a rescue method in a multitrauma patient combining pulmonary contusion and nonoperative internal bleeding: a case report and literature review. World J Emerg Surg 2015;10:15.

22. Moore SA, Dietl CA, Coleman DM. Extracorporeal life support during pregnancy. J Thorac Cardiovasc Surg 2016; 151:1154-60. 